\title{
Profiling of atmospheric turbulence strength and velocity using a generalised SCIDAR technique
}

\author{
V.A. Klückers, N.J. Wooder, T.W. Nicholls, M.J. Adcock, I. Munro, and J.C. Dainty \\ Applied Optics Group, Blackett Laboratory, Imperial College, London SW7 2BZ, UK
}

Received September 1; accepted November 13, 1997

\begin{abstract}
The measurement of the strength and velocity of atmospheric optical turbulence using a generalised SCIDAR technique is outlined and demonstrated. This method allows the full turbulent profile to be characterised from the telescope pupil up to any desired altitude. A number of example profiles from various astronomical observing sites are presented.
\end{abstract}

Key words: atmospheric effects - site testing telescopes

\section{Introduction}

The method described in this paper for remote turbulence profiling is based upon the SCIntillation Detection And Ranging (SCIDAR) technique which relies upon the analysis of crossed beam (i.e. binary star) scintillation patterns. This technique has been developed by Vernin and co-workers over a number of years (Vernin \& Roddier 1973; Rocca et al. 1974; Vernin \& Roddier 1975; Roddier \& Vernin 1977; Azouit et al. 1978; Vernin et al. 1979; Azouit \& Vernin 1980; Vernin \& Azouit 1983; Vernin et al. 1991) and more recently by Tyler (1992). A number of specific site investigations have been attempted using either SCIDAR alone (e.g. Racine \& Ellerbroek 1995) or in combination with temperature and pressure data obtained using balloon-borne instrumentation (Vernin et al. 1990; Vernin \& Muñoz-Tuñonn 1992, 1994). A number of spatial filtering techniques applied to scintillation signal detection from single stars have also been proposed for this particular problem (e.g. Ochs et al. 1976; Churnside et al. 1988; Zavorotny 1992). However, although the performance of SCIDAR is constrained by the availability of suitable binary star objects, one is able to obtain reliable profiles of a finer altitude sampling than that possible using spatial filtering methods on single stars.

Send offprint requests to: V.A. Klückers vince@op.ph.ic.ac.uk
With SCIDAR the strength of scintillation is used to estimate both the value of the atmospheric optical turbulence profile, $C_{n}^{2}(h)$, and the turbulence velocity, $V(h)$, as a function of height, $h$, using a triangulation technique. The methodology used here involves recording a large number of instantaneous scintillation patterns from a double star, calculating the average spatial and temporal correlation, and extracting the $C_{n}^{2}(h)$ profile using an integral inversion method and the $V(h)$ profile using template correlation.

Until very recently it was expected that such a method would be unable to characterise low altitude $(<5 \mathrm{~km})$ turbulence, thus requiring other techniques to be used in unison with SCIDAR to complete the profile information. However, as discussed later, the introduction of the concept of generalised SCIDAR by Fuchs et al. (1994), has shown that a simple modification of the basic SCIDAR method can allow this information to be recovered without this requirement, and here we show example results from our generalised SCIDAR experiments at various observing sites.

Although the profiling of optical turbulence and velocity is of great intrinsic interest in terms of atmospheric science, the main impetus for these experiments has been to obtain real $C_{n}^{2}(h)$ and $V(h)$ data for our theoretical work in the study of adaptive optics (AO) systems for astronomy.

The main concern of optimising traditional astronomical observations at a particular observing site has been with respect to the angular extent of the seeing disc observed at ground level, $\Phi$, which can be expressed simply as,

$\Phi \sim \lambda / r_{0}$

where $r_{0}$ is the turbulence coherence length given by,

$r_{0}=\left[0.42 k^{2} \cos ^{-1}(z) \int C_{n}^{2}(h) \mathrm{d} h\right]^{-3 / 5}$,

$k$ is the wavenumber and $z$ is the zenith angle of observation. Since $\Phi$ can be measured directly using long exposure imaging or indirectly using differential image motion (DIMM) methods (Martin 1987), explicit knowledge 
of the $C_{n}^{2}(h)$ profile itself is unnecessary for this purpose. However, in the design of an $\mathrm{AO}$ system for a particular astronomical observing site, not only does one require some knowledge of the statistics of the size of the seeing disc, but also that of the turbulence and velocity profile with height so that one may have some expectation of the detrimental effects of anisoplanatism. For example, for Angular Anisoplanatism (AA), the decorrelation with angle $\phi$ between the science object wavefront and the measured wavefront can be quantified in terms of the mean squared error between the two wavefronts, such that

$\sigma_{\mathrm{AA}}^{2}=\left(\phi / \phi_{0}\right)^{5 / 3}$

where $\phi_{0}$, the isoplanatic angle, is given by,

$\phi_{0}=\left[2.9 k^{2} \cos ^{-1}(z) \int C_{n}^{2}(h) h^{5 / 3} \mathrm{~d} h\right]^{-3 / 5}$.

Similarly, for an AO system guided by an artificially produced laser beacon, the decorrelation between the science object wavefront and the wavefront sampled by the beacon which leads to Focal Anisoplanatism (FA), can also be quantified in terms of $C_{n}^{2}(h)$ such that the mean squared error between the two wavefronts is given by,

$\sigma_{\mathrm{FA}}^{2}=\left(D / d_{0}\right)^{5 / 3}$

where $D$ is the telescope diameter and $d_{0}$ can be interpreted as an measure of the effective diameter of the AO imaging system when a single laser beacon is being used and can be approximated as,

$d_{0} \simeq\left[k^{2} \cos ^{-1}(z) \int_{0}^{H} C_{n}^{2}(h)(h / H)^{5 / 3} \mathrm{~d} h\right]^{-3 / 5}$,

where $H$ is the laser beacon altitude.

Knowledge of the velocity profile of the turbulent layers becomes useful when one considers the effect of Temporal Anisoplanatism (TA) in the AO corrected image due to the finite dwell time of the system between measuring the guide star wavefront and correcting the science object wavefront, $\tau_{\mathrm{d}}$. Once again, this can be quantified in terms of the mean squared error between the two wavefronts such that,

$\sigma_{\mathrm{TA}}^{2}=\left(\tau_{\mathrm{d}} / \tau_{0}\right)^{5 / 3}$

where $\tau_{0}$ is given in terms of both $V(h)$ and $C_{n}^{2}(h)$ as,

$\tau_{0}=\left[2.9 k^{2} \int C_{n}^{2}(h) V(h)^{5 / 3} \mathrm{~d} h\right]^{-3 / 5}$.

Consequently if one has knowledge of both $C_{n}^{2}(h)$ and $V(h)$, then all of the parameters which describe the performance of an $\mathrm{AO}$ system can be obtained.

In the future one could also envisage a real-time SCIDAR system being available to allow anisoplanatic effects to be quantified during an AO observing run, thus enabling image quality diagnostics to be made and provide information for reliable point spread function deconvolution if deemed necessary.

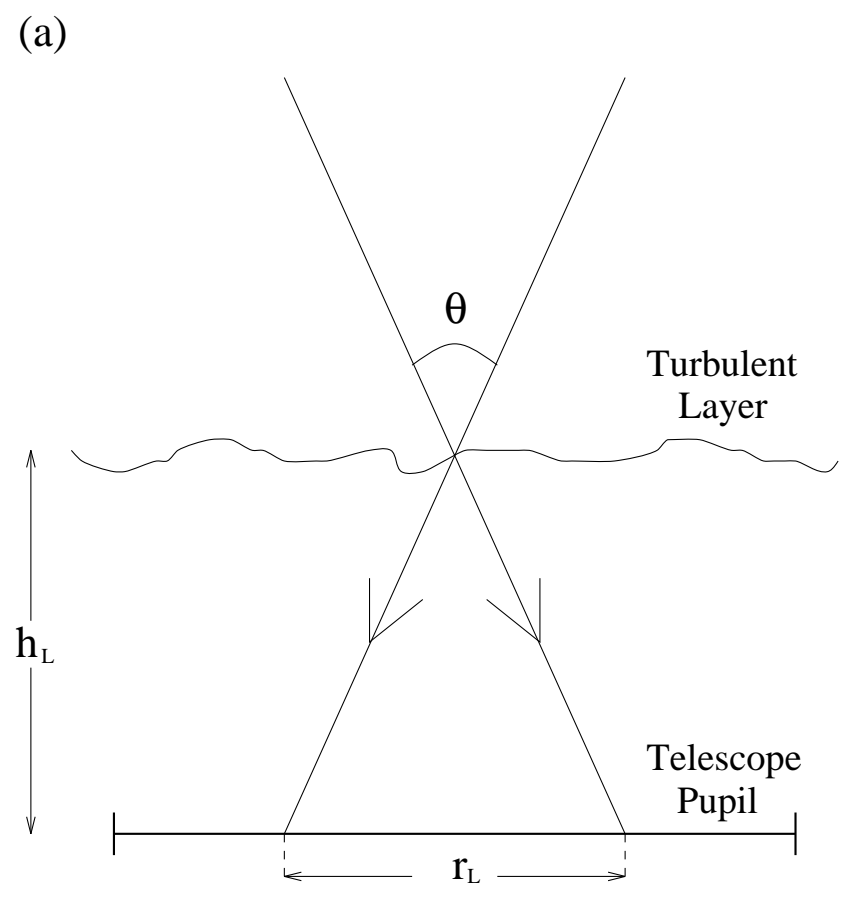

(b)

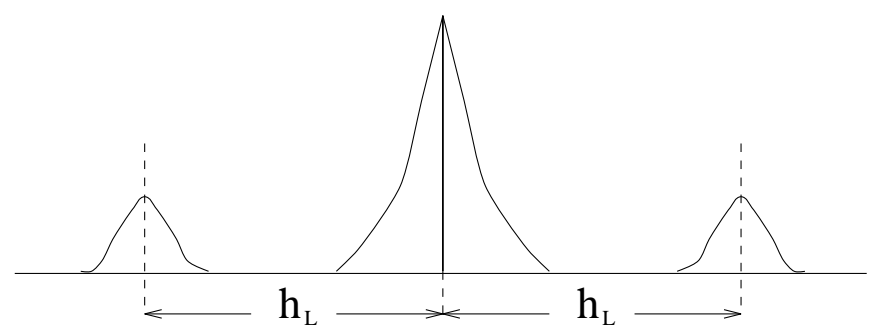

Fig. 1. A simplified one-dimensional representation of the SCIDAR technique for a single turbulent layer at height $h_{\mathrm{L}}$. a) A large number of short exposure images of the scintillation pattern due to a binary star (of angular separation $\theta$ ) are collected. b) The average auto-correlation of these data is calculated, from which the altitude of the turbulent layer above the telescope pupil is given simply by $h_{\mathrm{L}}=r_{\mathrm{L}} / \theta$

\section{Outline of methodology}

\subsection{Outline of the SCIDAR technique}

The SCIDAR technique is based upon calculating the average correlation of a large number of short exposure images of the scintillation pattern within a telescope pupil produced by a binary star. Figure 1 shows that in a simplified one-dimensional representation of the observing procedure for a binary object observed at zenith (a), a peak in the correlation plane occurs at a pupil separation $r_{\mathrm{L}}$, (b), and thus by simple geometry, denotes the presence of 
(a) Pupil Plane SCIDAR

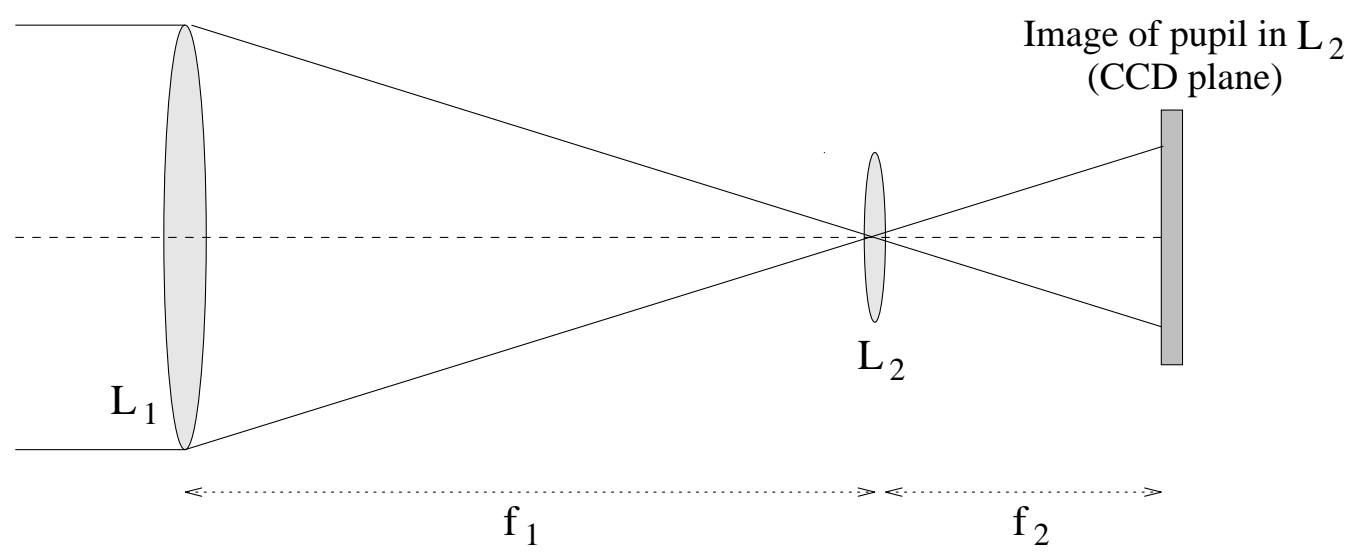

(b) Generalised SCIDAR

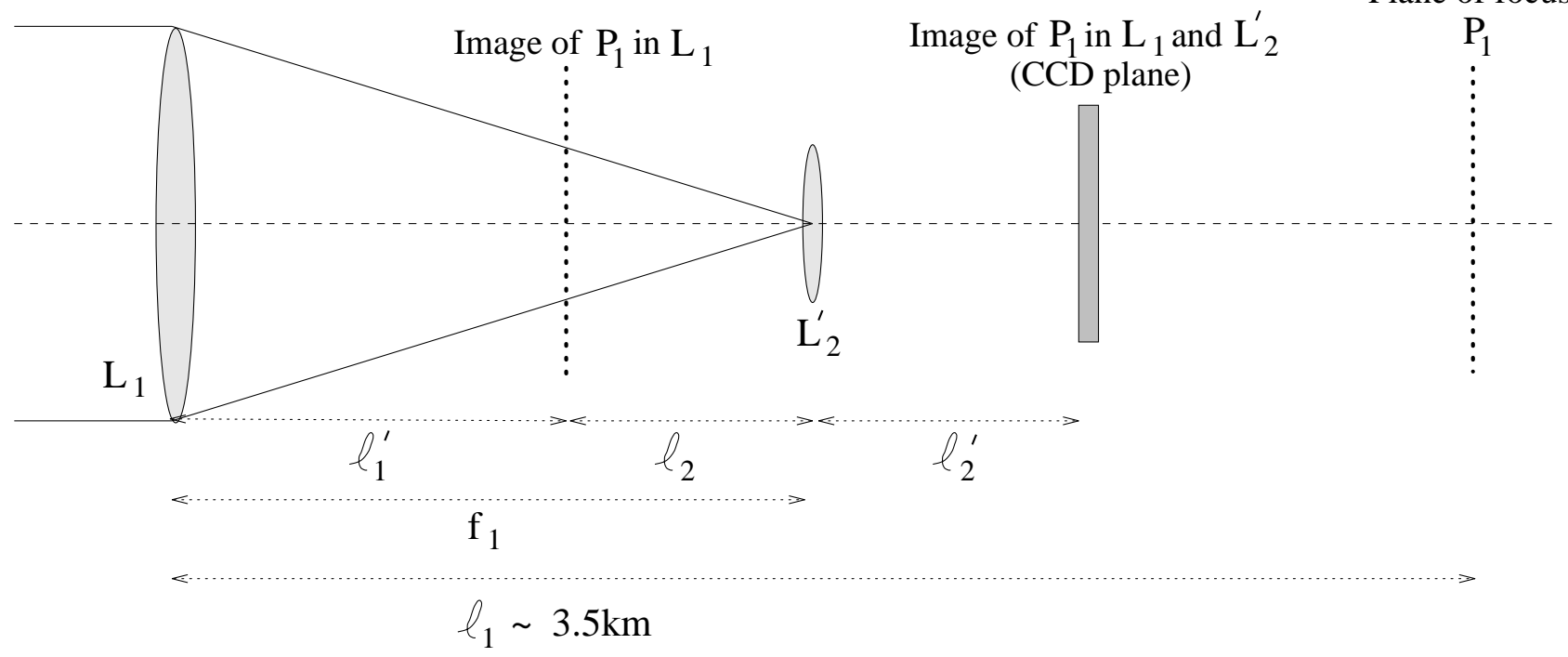

Fig. 2. Schematic diagram of the optical system for SCIDAR. a) shows the optical system for pupil plane SCIDAR. In this mode the telescope entrance pupil is imaged by the telescope mirror system $L_{1}$ and the field lens $L_{2}$. The focal length of the lens $L_{2}$ is chosen to give the correct image size at the detector plane. The image is effectively at infinity so that the final pupil image is formed at approximately the focal distance $f_{2}$ behind $L_{2} . \mathbf{b}$ ) shows the optical system for generalised SCIDAR. At the CCD plane this system forms a pupil image from a plane located the required distance behind the telescope mirror $\left(\ell_{1}\right)$. For this case then, the telescope mirrors $\left(L_{1}\right)$ form a virtual image of the plane of focus $\left(P_{1}\right)$ at a distance $\ell_{1}^{\prime}$ behind the telescope mirror. The new field lens $L_{2}^{\prime}$ re-images this virtual object to form the pupil image at the CCD plane. For example, for the JKT at La Palma; the telescope has a $1 \mathrm{~m}$ pupil with a F/15 focal ratio. A $159 \mathrm{~mm}$ field lens $L_{2}$ is required to give the correct image size for pupil plane SCIDAR. For Generalised SCIDAR the field lens $L_{2}$ is replaced by a lens with a focal length of $46 \mathrm{~mm}\left(L_{2}^{\prime}\right)$, still located at $159 \mathrm{~mm}$ from the CCD plane. A virtual image of a plane at $3.5 \mathrm{~km}$ is formed $\ell_{2} \simeq 64 \mathrm{~mm}$ in front of $L_{2}^{\prime}$ and this virtual object is imaged by $L_{2}^{\prime}$ onto the CCD detector

a single turbulent layer at an altitude $h_{\mathrm{L}}=r_{\mathrm{L}} / \theta$ above the telescope pupil. This simple situation can be generalised to the case where multiple turbulent layers exist, each one having an associated altitude calculable from the peak position within the average correlation. Also for the general case where the binary star is observed at some zenith angle $z$, then the geometrical conversion above becomes $h=r /(\theta \sec (z))$.
In the conventional operation of SCIDAR, the conjugate plane of the imaging system is placed at the altitude of the pupil of the telescope - referred to here as pupil-plane SCIDAR. However, low altitude turbulence cannot be determined in this mode since the scintillation requires a large distance over which to develop a signal strong enough to be detected. Consequently the correlation signal close to zero altitude is occupied only by a central core corresponding to the sum of all other higher 


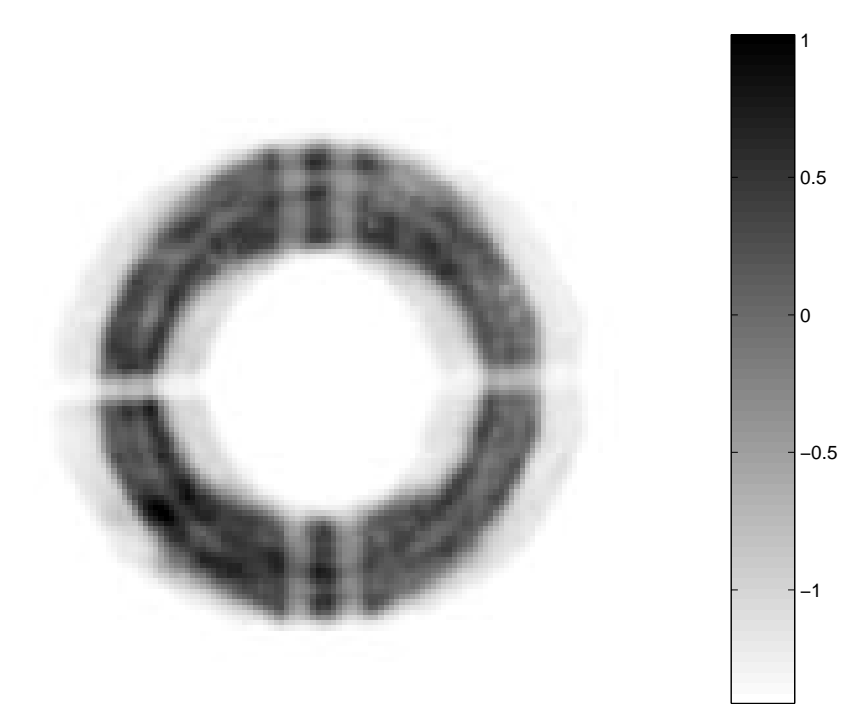

Fig. 3. Example average scintillation pattern image obtained in the generalised SCIDAR mode at the JKT, in La Palma from $180001.6 \mathrm{~ms}$ exposure frames. The scale is in relative intensity units

altitude peaks. This is shown diagrammatically as the central peak in Fig. 1a. Recently, however, a solution to this problem was proposed by Fuchs et al. (1994) in which one changes the conjugate height of the imaging system from that of the telescope pupil, to one some distance below it. In effect, this creates an extra distance for the scintillation pattern to develop to a detectable level and also shifts the central core to below the pupil (zero) altitude. Experiments performed by us in this generalised SCIDAR mode appear to confirm the validity of this approach and the results are discussed later in Sect. 4.

\subsection{Experimental setup}

The optical system used to acquire the SCIDAR data is shown schematically in Fig. 2. The imaging system consists of a GenIII image intensifier lens-coupled to a high speed $128 \times 128$ pixel CCD camera (DALSA CA-D10128A). The lens-coupling demagnifies, by a factor of approximately 5.2 , the intensifier output phosphor image onto the $2 \mathrm{~mm} \times 2 \mathrm{~mm}(16 \mu \mathrm{m}$ pixel $) \mathrm{CCD}$ chip. In Fig. $2 \mathrm{a}$, the pupil plane mode, the telescope entrance pupil is imaged onto the intensifier by the field lens $\left(L_{2}\right)$. The focal length of this lens is chosen such that the final CCD pixel size corresponds to approximately $1 \mathrm{~cm} \times 1 \mathrm{~cm}$ at the telescope entrance pupil. This choice of pixel sampling is made as a compromise between the received intensity per pixel and the detectability of atmospheric scintillation (see Sect. $3.1)$.

For example, for the Jacobus Kapteyn Telescope (JKT) in La Palma, the telescope has a $1 \mathrm{~m}$ pupil with an $\mathrm{F} / 15$ focal ratio and therefore an ideal focal length for the field lens $\left(L_{2}\right)$, to give this required sampling, would be $159 \mathrm{~mm}$. This would produce a $10.6 \mathrm{~mm}$ diameter pupil image at the intensifier, or equivalently a $2.04 \mathrm{~mm}$ pupil image on the CCD.

For the generalised mode shown in Fig. 2b, the field lens $\left(L_{2}\right)$ is replaced by one of a different focal length, $L_{2}^{\prime}$. The separation of field lens position to intensifier is unchanged however and thus the pupil plane sampling remains unchanged also. Using the thin lens equation one can calculate the required focal length for this generalised field lens for any chosen height of generalisation. For our measurements we are primarily concerned with ascertaining the strength and velocity of all turbulence present above the telescope altitude by conjugating to altitudes below that of the telescope pupil. Therefore for example, again for the JKT, in order to conjugate to a distance of $3.5 \mathrm{~km}$, say, then a field lens of $46 \mathrm{~mm}$ focal length is required.

The digital output from the DALSA Camera is acquired by a Bitflow Raptor PCI frame capture board controlled by a fast PC and the data is stored directly onto Digital Linear Tape. The frame exposure times normally used are $1.6 \mathrm{~ms}$ and $2.7 \mathrm{~ms}$, with frame acquisition rates of 305 and 188 frames per second respectively.

Figure 3 shows an example of the average scintillation pattern of 18000 frames obtained using this system on the JKT whilst running in the generalised SCIDAR mode. The sheared image of the telescope pupil is clearly visible.

\subsection{Data analysis}

\subsubsection{Determination of $C_{n}^{2}(h)$}

Prior to calculation of the $C_{n}^{2}(h)$ profile, each $i^{\text {th }}$ scintillation pattern image, $I_{i}(x, y)$, is first mean-normalised to obtain the relative intensity,

$I^{\prime}{ }_{i}(x, y)=\frac{I_{i}(x, y)-<I(x, y)>}{<I(x, y)>}$

(where $\langle I(x, y)\rangle$ is the average image) and the average auto-correlation of all such images calculated. To prevent the suppression of signal within the correlation plane for large separations within the pupil, this is then divided by the auto-correlation of an artificial pupil function (i.e. the optical transfer function of the pupil). This step is particularly important if using data collected from a telescope with a large obstruction ratio, as is the case for the example shown in Fig. 3 (see Sect. 3.1). The resulting function thus represents the two-dimensional intensity covariance, $C_{I}(\underline{r})$. Figure 4 a shows an example of this obtained using the generalised SCIDAR mode at the JKT for an effective conjugate distance of $2.5 \mathrm{~km}$, which clearly indicates the presence of at least two distinct turbulent layers. Each of these can be identified by their individual "triple-peak" pattern in a direction parallel to that of the binary star; comprising of a central peak contribution 

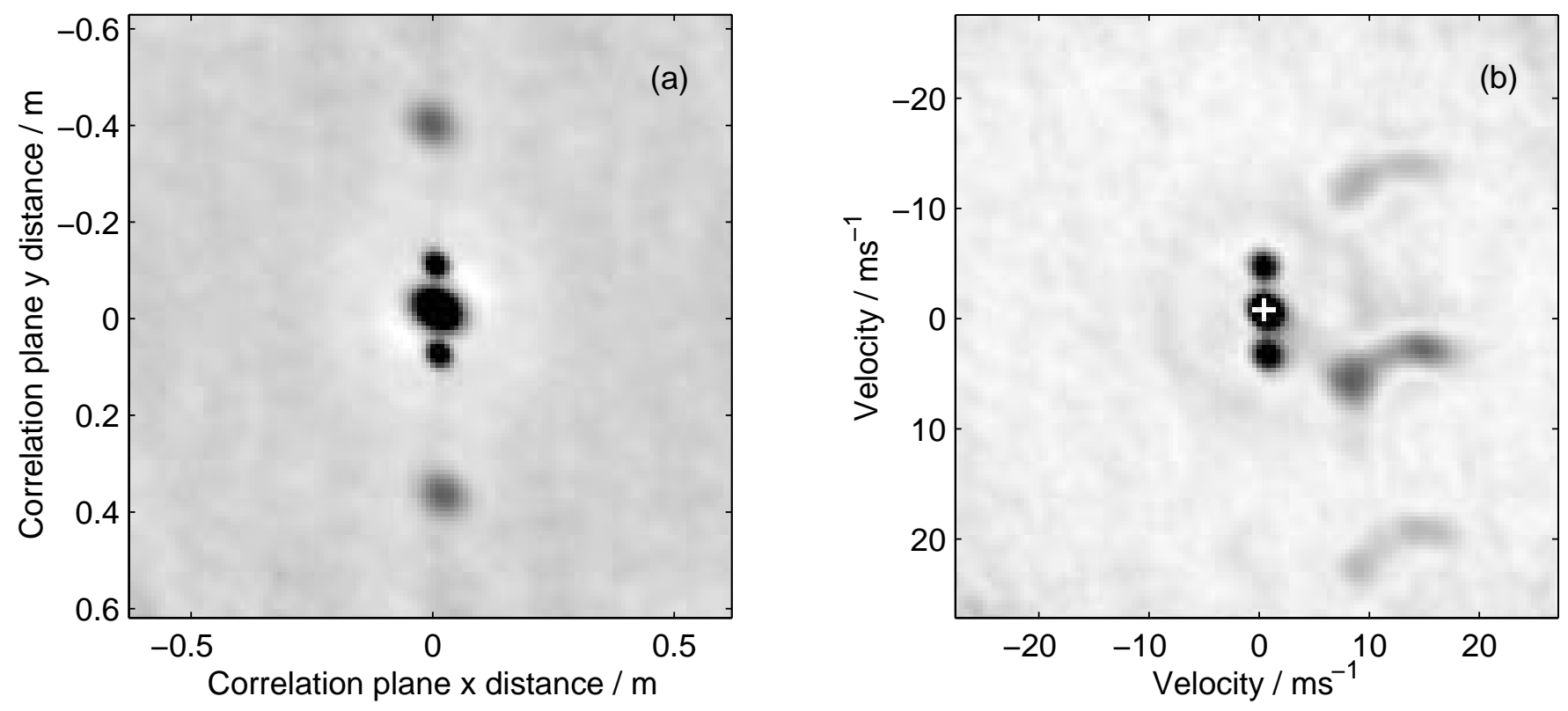

Fig. 4. Example a) spatial and b) temporal correlation plots generated from the same data set obtained from observations of the binary star $\gamma$ Aries (separation $7.8^{\prime \prime}$ and components of equal magnitude $4.8 m_{v}$ )

plus a (symmetrically-reflected) peak at a distance from the centre corresponding to the height of the layer above the altitude of conjugation via $h=r /(\theta \sec (z))$.

The finite width of these peaks results directly from the Fresnel length associated with each turbulent layer, given by $\Delta h_{\mathrm{F}}(h)=0.7 \sqrt{\lambda h} / \theta$ (Vernin \& Azouit 1983). This in turn effectively limits the degree of resolution in $C_{n}^{2}(h)$ attainable from the SCIDAR data. For the data collected thus far, $\Delta h_{\mathrm{F}}(h)$ ranges from around $700 \mathrm{~m}$ (for a pupil-conjugate distance of say $3 \mathrm{~km}$ ) to around $2000 \mathrm{~m}$ (for the maximum altitudes considered). Assuming a pupil plane sampling, $\Delta r$, of $1 \mathrm{~cm} \mathrm{pix}^{-1}$ and binary star separations between $4-9^{\prime \prime}$, then the altitude sampling available, given by $\Delta h=\Delta r /(\theta \sec (z))$, corresponds to around $200-500 \mathrm{~m}$, and thus represents a reasonable sampling rate of the Fresnel length at all altitudes.

A slice taken through the correlation plane, parallel to the direction of the binary star, $C_{\mathrm{I}}(r, \theta)$, can then be expressed as (Vernin \& Roddier 1975),

$$
\begin{aligned}
C_{\mathrm{I}}(r, \theta) & =\left(\frac{B_{1}^{2}+B_{2}^{2}}{\left(B_{1}+B_{2}\right)^{2}}\right) A_{\mathrm{I}}(r, 0) \\
& +\left(\frac{B_{1} B_{2}}{\left(B_{1}+B_{2}\right)^{2}}\right)\left[A_{\mathrm{I}}(r, \theta)+A_{\mathrm{I}}(r,-\theta)\right]
\end{aligned}
$$

where $B_{1}$ and $B_{2}$ are the relative brightnesses of the binary star components, $A_{\mathrm{I}}(r, 0)$ represents the central (single star) correlation peak and where $A_{\mathrm{I}}(r, \pm \theta)$ contains the information that is required regarding the strength and position of the turbulent layers.
The log amplitude covariance of the scintillation fluctuations then follows as,

$A_{\chi}(r, \theta)=\frac{\ln \left[A_{\mathrm{I}}(r, \theta)+1\right]}{4}$,

and finally, if one can assume both the Rytov approximation (i.e. that $A_{\chi}(0)=\sigma_{\chi}^{2}<0.3$, where $\sigma_{\chi}^{2}$ is the variance of the intensity fluctuations within a single pixel) and Kolmogorov statistics for the optical turbulence fluctuations, then it can be shown that this function can be expressed in terms of $C_{n}^{2}(h)$ as (Tyler 1992),

$A_{\chi}(r, \theta)=\frac{8.16 k^{2}}{4 \pi} \int_{0}^{\infty} C_{n}^{2}(h) h^{5 / 6} F(Q) \mathrm{d} h$,

where,

$F(Q)=\int_{0}^{\infty} K^{-8 / 3} J_{0}(Q K)\left[1-\cos \left(K^{2}\right)\right] \mathrm{d} K$,

and

$Q=\left[\frac{k}{h \sec (z)}\right]^{1 / 2}|r-\theta h \sec (z)|$.

Therefore, given the known quantities (13) and (14) one is required to calculate $C_{n}^{2}(h)$ from Eq. (12) - a classic inversion problem (specifically, a Fredholm integral of the first kind).

The particular method one might employ for this problem (and also to some extent the confidence of the solution) depends very much upon the $C_{n}^{2}(h)$ altitude sampling that is required. Tests performed upon a number of high resolution $C_{n}^{2}(h)$ profiles obtained by balloon-borne instruments (Vernin \& Muñoz-Tuñôn 1992) have shown 

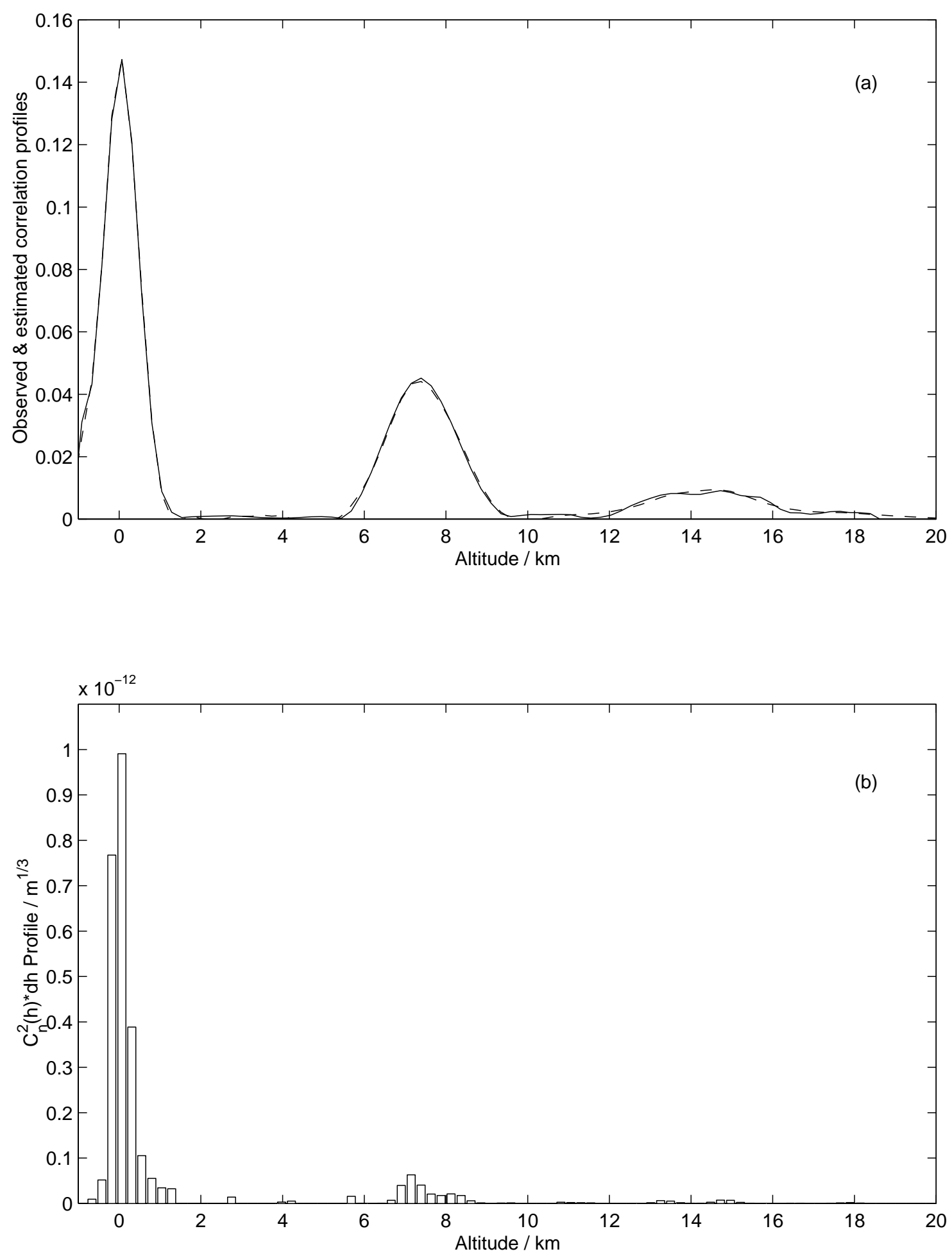

Fig. 5. a) An example 2-d average cross-correlation plot and b) the corresponding $C_{n}^{2}(h)$ profile calculated using a maximum entropy based method. Note that the apparent single turbulent layer centred at $7.75 \mathrm{~km}$ in a) has been resolved into two layers in b). The resolution of the inversion calculation is $300 \mathrm{~m}$ 
that the main parameters which determine AO anisoplanatic performance can be calculated with reasonable accuracy provided that the altitude sampling, $\Delta h$, is less than $1 \mathrm{~km}$. For the data collected thus far, the altitude resolution available is between around $200-500 \mathrm{~m}$, and therefore comfortably within this constraint. However it has been found that a simple least-squares inversion of Eq. (12) for these data is incapable of producing a reliable solution unless one rectangularises the two-dimensional integral equation such that multiple pupil samples map onto each altitude sample and $\Delta r$ remains fixed at $1 \mathrm{~cm} \mathrm{pix}{ }^{-1}$ but $\Delta h$ is extended to $2-3 \mathrm{~km}$.

Although, ultimately, it is the Fresnel length that limits the altitude resolution attainable, in an effort to realise the altitude sampling that is made available by that within the pupil, a number of iterative inversion methods have been explored. One that has proved successful is based upon a maximum entropy routine which also allows for the controlled propagation of noise between the data and solution (Skilling \& Bryan 1984). In practice, we first calculate a crude (large $\Delta h$ ) least-squares inversion of $C_{n}^{2}(h)$ using a rectangularised version of Eq. (12) and then use an interpolated version of this as the first guess estimate for the iterative routine. As an aid to deciding when the routine should be terminated, the following RMS difference error metric is calculated following each iteration:

$\mathcal{E}_{\mathrm{A}}=\sqrt{\frac{\left\langle\left(A_{\text {data }}(r, \theta)-A_{\text {best }-\mathrm{fit}}(r, \theta)\right)^{2}>\right.}{<A_{\text {data }}^{2}(r, \theta)>}}$,

where, $A_{\text {data }}(r, \theta)$ is the observed one-dimensional slice through the correlation plane and $A_{\text {best-fit }}(r, \theta)$ is the function calculated using the current (best-fit) solution to $C_{n}^{2}(h)$ from Eq. (12).

In Fig. 5, (a) shows the correctly scaled correlation slice, $A_{\text {data }}(r, \theta)$, of Fig. $4 \mathrm{a}$, and in (b) the corresponding $C_{n}^{2}(h)$ profile obtained. Also shown in Fig. 5a, for comparison is $A_{\text {best-fit }}(r, \theta)$ which gives some indication of the accuracy of the solution obtained where, in this case, $\mathcal{E}_{\mathrm{A}}=4.3 \%$. It is interesting to note that for the particular example shown here the apparent single correlation peak at a height of $7.5 \mathrm{~km}$ in Fig. 5a has been resolved into two distinct turbulent layers within the corresponding $C_{n}^{2}(h)$ profile of Fig. $5 \mathrm{~b}$ at heights of $7.2 \mathrm{~km}$ and $8.1 \mathrm{~km}$.

The apparent width of the dominant turbulent layers shown here probably does not reflect the true physical depth over which the optical turbulence exists. It is more likely that this apparent spread of $C_{n}^{2}(h)$ values is due to the effective resolution imposed by the Fresnel length, as discussed in Sect. 2.3.1. Therefore, although it appears that one is oversampling each peak in the $C_{n}^{2}(h)$ profile, the suspicion is that in fact the layers of turbulence themselves could be substantially thinner.

Confidence in the inversion methods used has been obtained by testing them on realistic simulated SCIDAR data generated using a Fresnel wavefront propagation code developed within our group (Adcock et al. 1996). Figure 6 gives one such result, which shows a typical simulated correlation image used in these tests and compares the true $C_{n}^{2}(h)$ input data with the solution obtained. Although the blurring effect of the Fresnel length is clearly apparent, the integrated values of $C_{n}^{2}(h)$ for each layer agree to within a few percent.

\subsubsection{Determination of $V(h)$}

Prior to calculation of the velocity profile, $V(h)$, the same initial processing steps are taken as for the $C_{n}^{2}(h)$ except that now the average cross-correlation of consecutive mean-normalised images is calculated, each separated by a time interval $\Delta t$. The resulting two-dimensional function thus represents the average spatio-temporal correlation of the turbulence, the sampling interval of which is given simply by $\Delta V=\Delta r / \Delta t$.

Figure $4 \mathrm{~b}$ shows such a plot generated from the same data as in Fig. 4a, with $\Delta t=23 \mathrm{~ms}$ (corresponding to $\Delta V=0.42 \mathrm{~ms}^{-1}$ ). The zero velocity position within this plot is denoted at the centre by a cross. In comparing this with the spatial-correlation plot of Fig. 4a one can see that, as indicated by the the $C_{n}^{2}(h)$ of Fig. $5 \mathrm{~b}$, the apparent single peak at the greatest distance from the central core has been resolved into two layers at heights of $7.2 \mathrm{~km}$ and $8.1 \mathrm{~km}$ with associated velocities of $14.6 \mathrm{~ms}^{-1}$ (wind direction Position Angle, $\mathrm{PA}=103^{\circ}$ ) and $9.8 \mathrm{~ms}^{-1}$ $\left(\mathrm{PA}=130^{\circ}\right)$ respectively. It is also worth noting that the correlation peak closest to the central core (corresponding to a height close to the telescope pupil) has shifted only very slightly from its central position in the spatialcorrelation $\left(V=0.01 \mathrm{~ms}^{-1}\right)$. This is indicative perhaps of the presence of almost static turbulence within the telescope dome since the ground wind speed recorded at this time was $13 \mathrm{~ms}^{-1}$.

The algorithm to allow automative calculation of $V(h)$ is based upon the assumption that the optical turbulence is highly discretised (the justification for this is clarified later in Sect. 4). Having first determined the altitudes of each discrete layer and the orientation of the binary star observed, a template is then constructed for each layer, each one representing their individual spatial correlation signal. The cross-correlation of each of these templates with the spatio-temporal plot then allows the speed and direction of each layer to be identified.

\section{Practical considerations}

It is now worthwhile to highlight a number of practical considerations with respect to the actual implementation of the SCIDAR technique. 

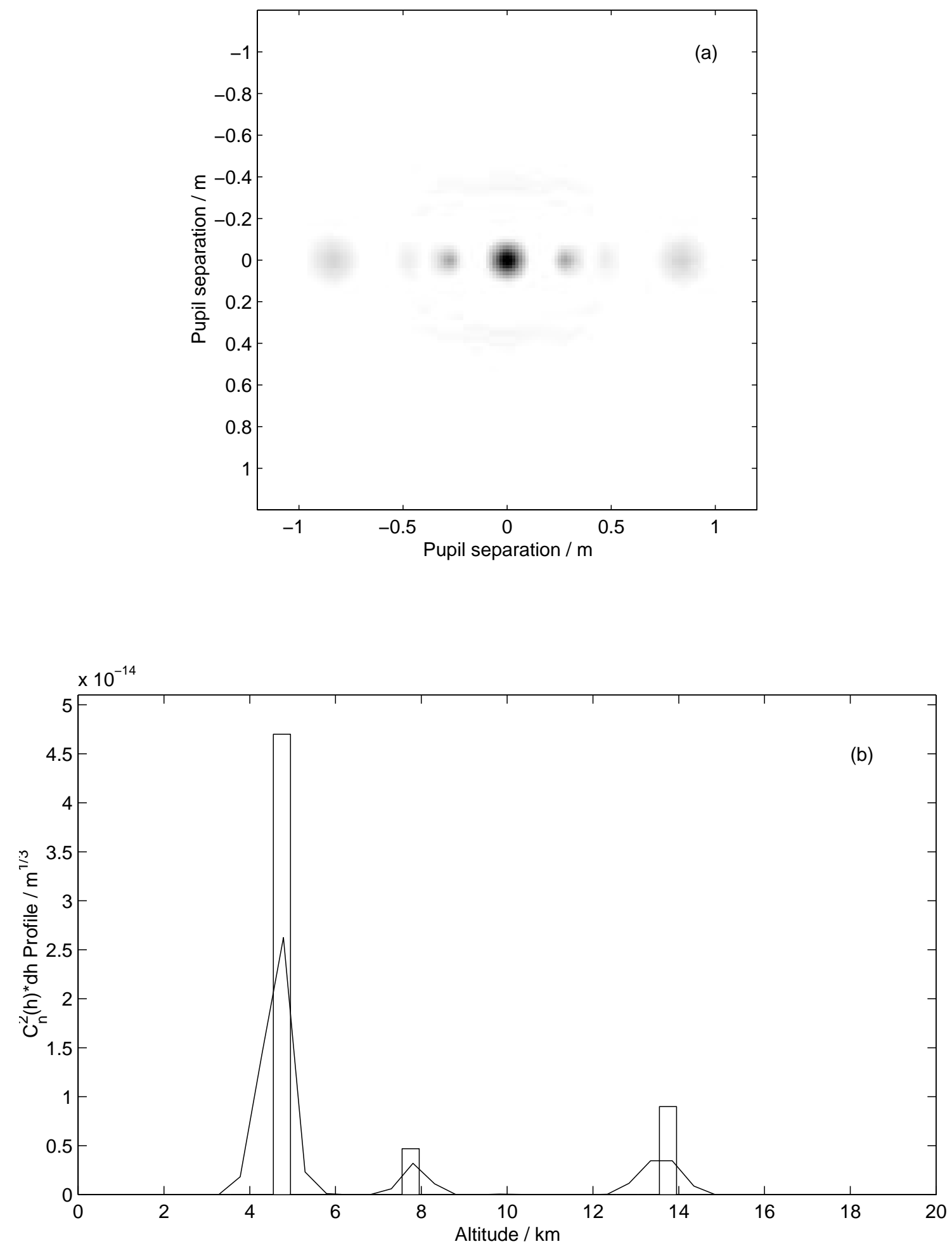

Fig. 6. a) Simulated spatial correlation data along with b) a comparison of the model input $C_{n}^{2}(h)$ profile (bar-plot) and that obtained using our integral inversion method 


\subsection{Effect of finite spatial and temporal sampling}

The theory outlined in Sect. 2.3 assumes an infinitely small spatial and temporal scintillation detection sampling rate. Obviously, however, for the practical implementation of SCIDAR one has to compromise the level of detectable scintillation for the sake of obtaining a reasonably high signal-to-noise level in the detected intensity. Consequently our choice of spatial and temporal sampling means that the level of scintillation detected will be less than that inferred by the theory, leading to an underestimate of $C_{n}^{2}(h)$. This may explain, for example, some of the relatively high values of $r_{0}$ we obtain - as shown in Sect. 4. For the relatively low sampling rates used in our system $\left(\Delta r \simeq 1 \mathrm{~cm} \mathrm{pix}^{-1}\right.$ and $\Delta t=1.6$ or $\left.2.7 \mathrm{~ms}\right)$ this underestimate could be corrected, to first order, by linearly-calibrating our results with respect to the integrated $C_{n}^{2}(h)$ value determined by other means during the observations. This feasibility is planned for our purposebuilt SCIDAR system currently under construction.

\subsection{Signal-to-noise considerations}

At any particular time during an observation run a number of binary star objects may be available for the collection of SCIDAR data. Therefore an obvious consideration one first has to make is regarding the signal-to-noise tradeoff between various potential targets. Given the magnitudes of each component for a number of potential targets, one can calculate the error propagation for the expression involving $B_{1}$ and $B_{1}$ in the second term of Eq. (10) to predict the relative average signal-to-noise ratio (SNR) with respect to photon noise. Figure 7 shows such a calculation for a typical range of values encountered in practice and enables one therefore to make an informed choice.

However, if one is collecting data across the whole telescope pupil using a telescope with a large obstruction ratio, one also has to take into account the effect of the pupil transfer function upon the SNR within the correlation plane. This is particularly so if one is operating in the generalised SCIDAR mode since one is then imaging the scintillation pattern viewed through two overlapping pupils. The degree of pupil overlap is directly proportional to both the angular separation of the binary and the effective distance below the telescope pupil one defocuses to. Figure 8 shows the predicted pupil overlap transfer function for various binary separations assuming a $1 \mathrm{~m}$ telescope pupil of obstruction ratio 0.3 and a typical defocus distance of $3.5 \mathrm{~km}$. As can be seen in the most extreme case, for example beyond separations of 8 arcseconds, the distorted transfer function can significantly reduce the SNR for high altitudes.

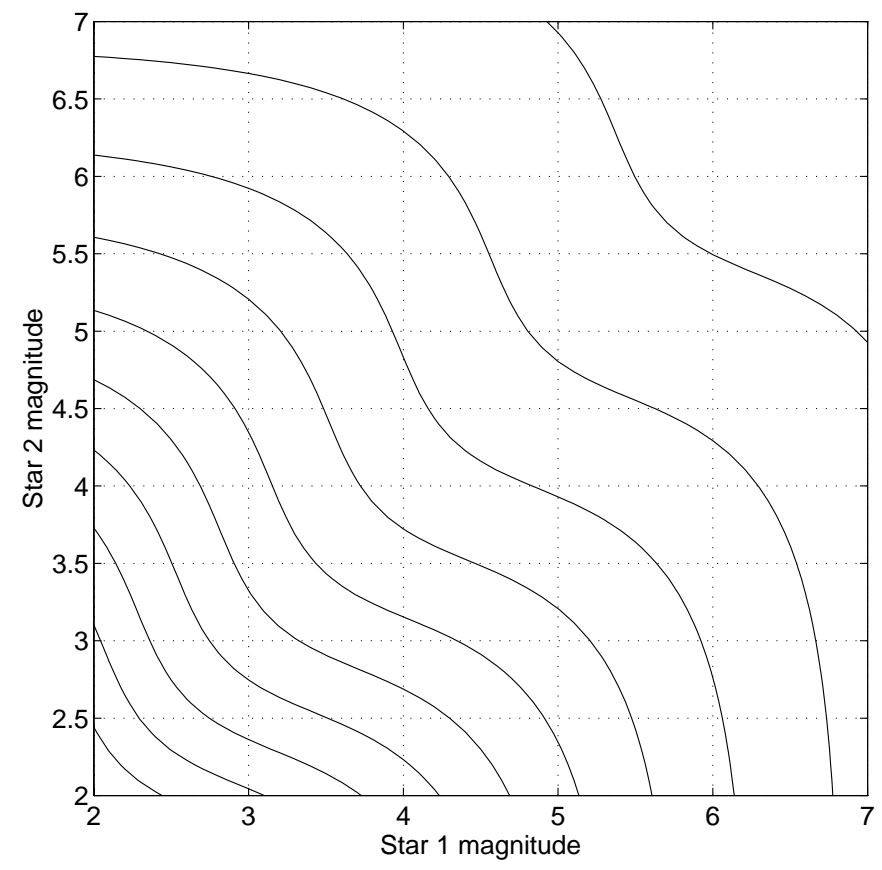

Fig. 7. Contour plot of the relative SNR for various binary star magnitude combinations. Contours are at $10 \%$ the peak level. The values decrease from left to right

\section{3. $C_{n}^{2}(h)$ altitude resolution}

Since close to zenith, $\Delta h=\Delta r / \theta$, in theory one is free to choose whatever altitude sampling length is required. However, for a particular telescope diameter $D$, in practice a restriction is imposed by the requirement that the maximum height attained should be greater than the sum of the height for which optical turbulence is assumed to be negligible and the magnitude of the generalised SCIDAR conjugate plane distance, $\left|h_{\mathrm{conj}}\right|$, ie: $h_{\max }=D / \theta \geq 20 \mathrm{~km}$ $+\left|h_{\text {conj }}\right|$.

These restrictions upon binary star separation are shown graphically in Fig. 9 for an assumed pupil sampling of $1 \mathrm{~cm} \mathrm{pix}^{-1}$, a telescope pupil diameter of $1 \mathrm{~m}$ and a conjugate plane distance of $3.5 \mathrm{~km}$. Taken in combination, the restrictions implied in Fig. 8 and Fig. 9 show that for this particular configuration, binary separations between around $4-7$ arcseconds are desirable.

It must be noted that both the upper limit upon $\theta$ and the SNR within the correlation plane can be increased, if using a telescope of a large enough diameter (e.g. $D>$ $3.5 \mathrm{~m}$ ) to enable an artificial circular pupil greater than $1 \mathrm{~m}$ to be imposed within the image of the segment of the mirror observed. In this case, assuming a similar pupil sampling as previously, the restriction upon $\theta$ then only originates from the size of the CCD, such that in our case $\theta<11^{\prime \prime}$. 


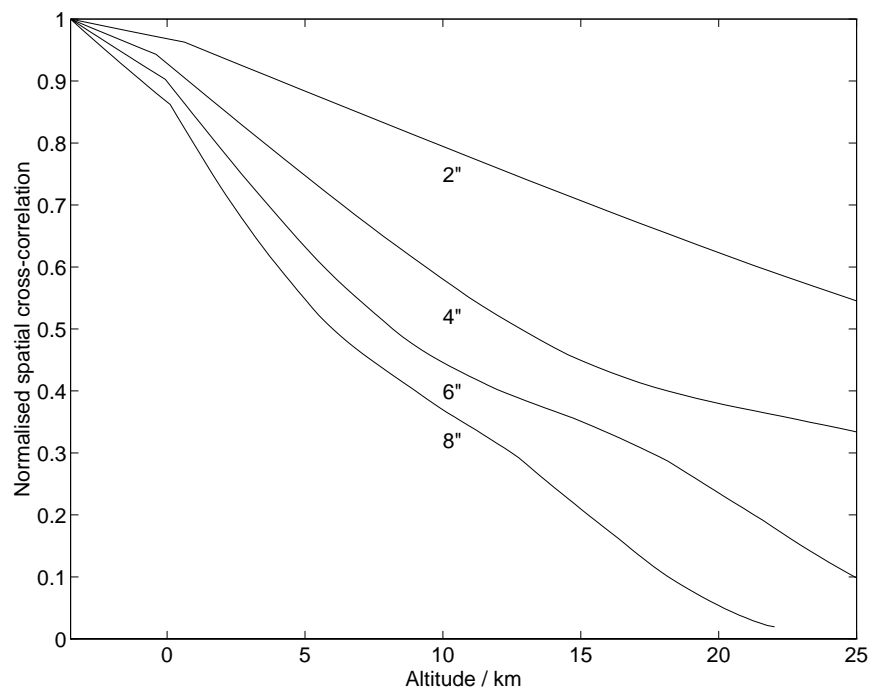

Fig. 8. Example transfer functions of overlapping pupils, for the generalised SCIDAR case, as a function of altitude. Curves are given for binary star separations of 2, 4, $6 \& 8$ arcseconds and assume a telescope diameter of $1 \mathrm{~m}$ and $1 \mathrm{~cm} \mathrm{pix}{ }^{-1}$ image plane sampling

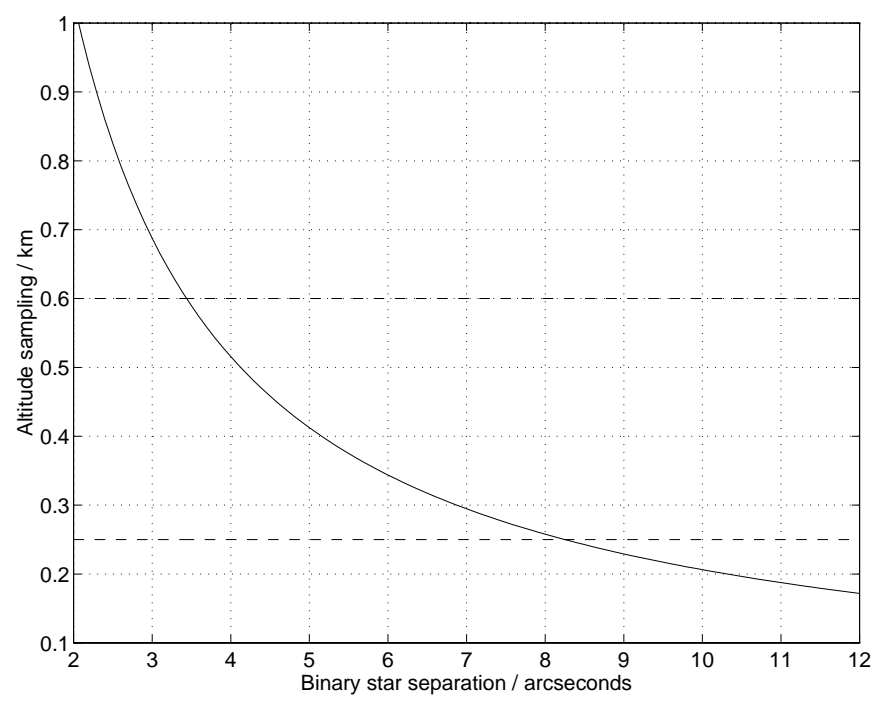

Fig. 9. Plot of altitude resolution as a function of binary separation showing the limits imposed for a reasonable altitude sampling (top) and a maximum altitude reached of $25 \mathrm{~km}$ above image plane (bottom), for a $1 \mathrm{~m}$ telescope assuming $1 \mathrm{~cm} \mathrm{pix}^{-1}$ image plane sampling

\subsection{Estimating the error on $C_{n}^{2}(h)$}

Having calculated a solution $C_{n}^{2}(h)$ profile from the data, one also requires some knowledge of the reliability of this solution. Although this is difficult to ascertain directly when using the iterative maximum entropy routine (Sect. 2) due to the non-linear nature of the iterative procedure, it has been found that it is possible to estimate the RMS error of a solution using a numerical approach.

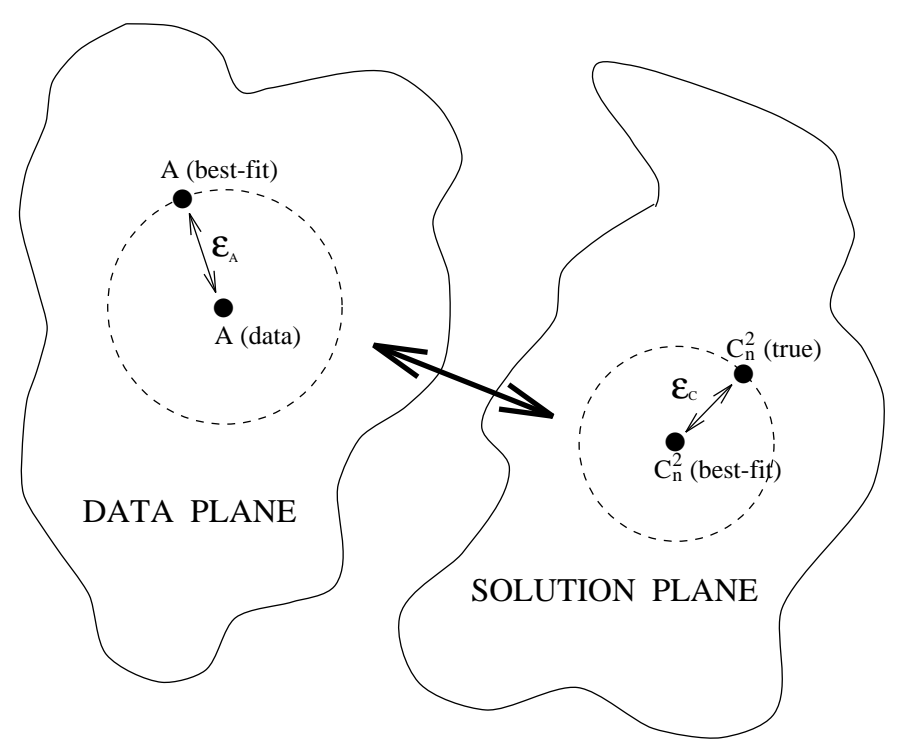

Fig. 10. Schematic description of the method used to estimate the RMS error on any particular $C_{n}^{2}(h)$ profile obtained

To enable this we define a "solution plane", which contains both the true (and unknown) $C_{n}^{2}$ true profile and the best-fit solution profile, $C_{n}^{2}$ best-fit, and a "data plane", within which resides both the true (observed) data, $A_{\text {data }}$, and the correlation function obtained from the $C_{n}^{2}$ best-fit profile, $A_{\text {best-fit }}$.

A schematic outline of this representation is shown in Fig. 10. Given that one can then represent the RMS difference $\mathcal{E}_{\mathrm{A}}$ given by Eq. (15), as the geometrical distance in the data plane between $A_{\text {data }}$ and the final $A_{\text {best-fit }}$, what one wishes to find is the corresponding distance between $C_{n \text { true }}^{2}$ and $C_{n \text { best-fit }}^{2}$, namely $\mathcal{E}_{\mathrm{C}}-$ as indicated in Fig. 10.

The approach taken here is to numerically perturb the $C_{n}^{2}$ best-fit profile about its position within the solution plane by incremental distances by applying random Gaussian noise, until the RMS distance within the data plane between its corresponding correlation function and $A_{\text {data }}$ is equal to the RMS distance $\mathcal{E}_{\mathrm{A}}$. When this occurs the RMS distance between $C_{n}^{2}$ best-fit and its perturbed value can then be considered as describing the radius of a circle within the solution plane upon which the "true" value of $C_{n}^{2}(h)$ lies and therefore represents a reasonable approximation of the RMS error $\mathcal{E}_{\mathrm{C}}$.

In doing so one is assuming that the solution $C_{n \text { best-fit }}^{2}$ is close to the global minimum within the solution plane, or at least within a local minimum which is not very different from the true solution. This assumption is justified both by the relatively low value of $\mathcal{E}_{\mathrm{A}}$ we achieve prior to termination of the iterative routine and also by the strongly linear relationship that has been found in all cases between $\mathcal{E}_{\mathrm{A}}$ and $\mathcal{E}_{\mathrm{C}}$ as the degree of perturbation applied to $C_{n \text { best-fit }}^{2}$ is increased. 
Table 1. Summary of the values of the Fried parameter $r_{0}$, isoplanatic angle $\phi_{0}$ and laser guide star parameter $d_{0}$, calculated from each of the three sets of example profiles for each of the observing sites shown in Fig. 11, along with the average $\tau_{0}$ value over this time interval

\begin{tabular}{|c|c|c|c|c|c|}
\hline & & $\overline{r_{0}(\mathrm{~m})}$ & $\overline{\phi_{0}\left({ }^{\prime \prime}\right)}$ & $d_{0}(\mathrm{~m})$ & $\overline{\tau_{0}(\mathrm{~ms})}$ \\
\hline La Palma & set 1 & 0.05 & 0.9 & 1.6 & \multirow{3}{*}{3.2} \\
\hline La Palma & set 2 & 0.05 & 0.9 & 1.5 & \\
\hline La Palma & set 3 & 0.04 & 0.7 & 1.2 & \\
\hline Siding Sprin & set 1 & 0.16 & 4.0 & 6.9 & \multirow{3}{*}{25.8} \\
\hline Siding Sprin & set 2 & 0.14 & 3.6 & 6.2 & \\
\hline Siding Sprin & set 3 & 0.15 & 4.7 & 7.8 & \\
\hline Calar Alto & set 1 & 0.19 & $\overline{5.5}$ & $\overline{9.2}$ & \multirow{3}{*}{29.9} \\
\hline Calar Alto & set 2 & 0.18 & 4.0 & 6.7 & \\
\hline Calar Alto & set 3 & 0.19 & 5.6 & 9.3 & \\
\hline
\end{tabular}

Having performed this calculation for a number of different $C_{n}^{2}(h)$ profiles, it has been found that the value of $\mathcal{E}_{\mathrm{C}}$ is greater than the value of $\mathcal{E}_{\mathrm{A}}$ in most cases. For example for the case shown in Fig. $5, \mathcal{E}_{\mathrm{A}}$ and $\mathcal{E}_{\mathrm{C}}$ are found to be $4.3 \%$ and $7.2 \%$ respectively. This highlights the need for high SNR data for a reliable $C_{n}^{2}(h)$ solution.

\section{Example results and discussion}

In this section we show example $C_{n}^{2}(h)$ and $V(h)$ profiles obtained from three astronomical observing sites using the generalised SCIDAR technique. It is intended that a full analysis of the findings from these data will follow in subsequent papers. Therefore, the results shown here are given as example cases only and are not meant to represent typical observing conditions.

The observing sites considered are:

Fig. 11a; La Palma, Canaries (JKT $1 \mathrm{~m}$ ) in November 1996, Fig. 11b; Siding Springs, Australia (40 inch) in January 1997, Fig. 11c; Calar Alto, Spain (2.2 m) in April 1997.

Each figure shows three consecutive $C_{n}^{2}(h)$ profiles, each calculated from a 7 second block of data (corresponding to 2000, $1.6 \mathrm{~ms}$ exposure, data frames) - each block being separated by around 10 seconds. For each figure, the first profile is shown as a solid line, the second as a dotted line and the third as a dot-dashed line. Using the methodology outlined in Sect. 3.4, the RMS error of each $C_{n}^{2}(h)$ profile shown is estimated to be within $6 \%$. Also shown within each figure is the average speed and PAdirection of each dominant turbulent layer over the time interval covered. Table 1 summarises the various $r_{0}, \phi_{0}$ and $d_{0}$ values calculated from each of the profiles, as well as the average $\tau_{0}$ value over the time interval considered. The $d_{0}$ parameter is calculated using the formulation of Tyler (1994) assuming a (Sodium) laser beacon altitude of $90 \mathrm{~km}$.

The three sets of profiles appear remarkably similar in both their general form and as such share a number of in- teresting characteristics. Firstly, each set of $C_{n}^{2}(h)$ profiles appear to be highly discretised. In fact this phenomena is seen in all of the $C_{n}^{2}(h)$ profiles we have calculated to date such that at any particular moment the optical turbulence appears to be dominated by a small number (perhaps 2-4) of thin layers. Because of the intrinsic SCIDAR resolution (Sect. 3.3), the actual thickness of these layers is difficult to ascertain. However in many cases the apparent thickness corresponds closely to that expected from the Fresnel size alone - suggesting that they could be much thinner than the $200-300 \mathrm{~m}$ altitude sampling used in these calculations. Also, in each case the turbulence is dominated strongly by slow moving, low altitude turbulence close to the telescope pupil. Static telescope mirror aberrations are excluded as a possible explanation of this phenomena since it is the covariance of the relative intensity fluctuations that are calculated. As discussed previously the source of this turbulence is most probably due to that inside the telescope dome (i.e: dome-seeing) since in each case the wind ground-speed was very much greater than the measured speed of the layer. Obviously some level of optical turbulence must exist within the boundary layer outside of the of dome. However it appears that in most cases it is difficult to discern its presence due to the dominating effect of the Fresnel-broadened dome-seeing layer.

A rapid variation in $C_{n}^{2}(h)$ far in excess of that expected due to noise alone is also noticeable from these results. The scale of these variations are a typical feature of all of the $C_{n}^{2}(h)$ profiles we have examined so far such that the strength of the optical turbulence due to any particular layer can fluctuate by as much as a factor of 2 or 3 within tens of seconds. One notable difference in the example profiles shown is that between the strength of those of the JKT in La Palma (a) and the others, and this is reflected in the parameters given in Table 1. Not only is the integrated $C_{n}^{2}(h)$ close to an order of magnitude greater but the speed of the dominant layer in the upper atmosphere is also very much larger.

Figure 12 illustrates the longer term changes in $C_{n}^{2}(h)$ that have been commonly observed. Shown here are three bar-plot profiles from the Siding Springs site taken during the same observing run as above. Each profile was calculated from a four minute block of data (thus allowing a higher SNR than previously) with each block separated in time by 40 minutes. As can be seen, the structure of turbulent layers at low altitudes remains reasonably consistant throughout this period, whereas at high altitudes this is not the case. The impact of these changes in layer structure upon angular and focal anisoplanatism is emphasised graphically in Fig. 13 where the same profiles are shown after scaling with $h^{5 / 3}$. Although the value of $r_{0}$ remains between 14.5 and $16.0 \mathrm{~cm}$, the value of $\phi_{0}$ and $d_{0}$ increase steadily from $3.0^{\prime \prime}$ to $5.0^{\prime \prime}$ and from $5.3 \mathrm{~m}$ to $8.8 \mathrm{~m}$ respectively. This figure also serves to emphasise again the high degree of discretisation that can occur within a turbulent atmosphere. 

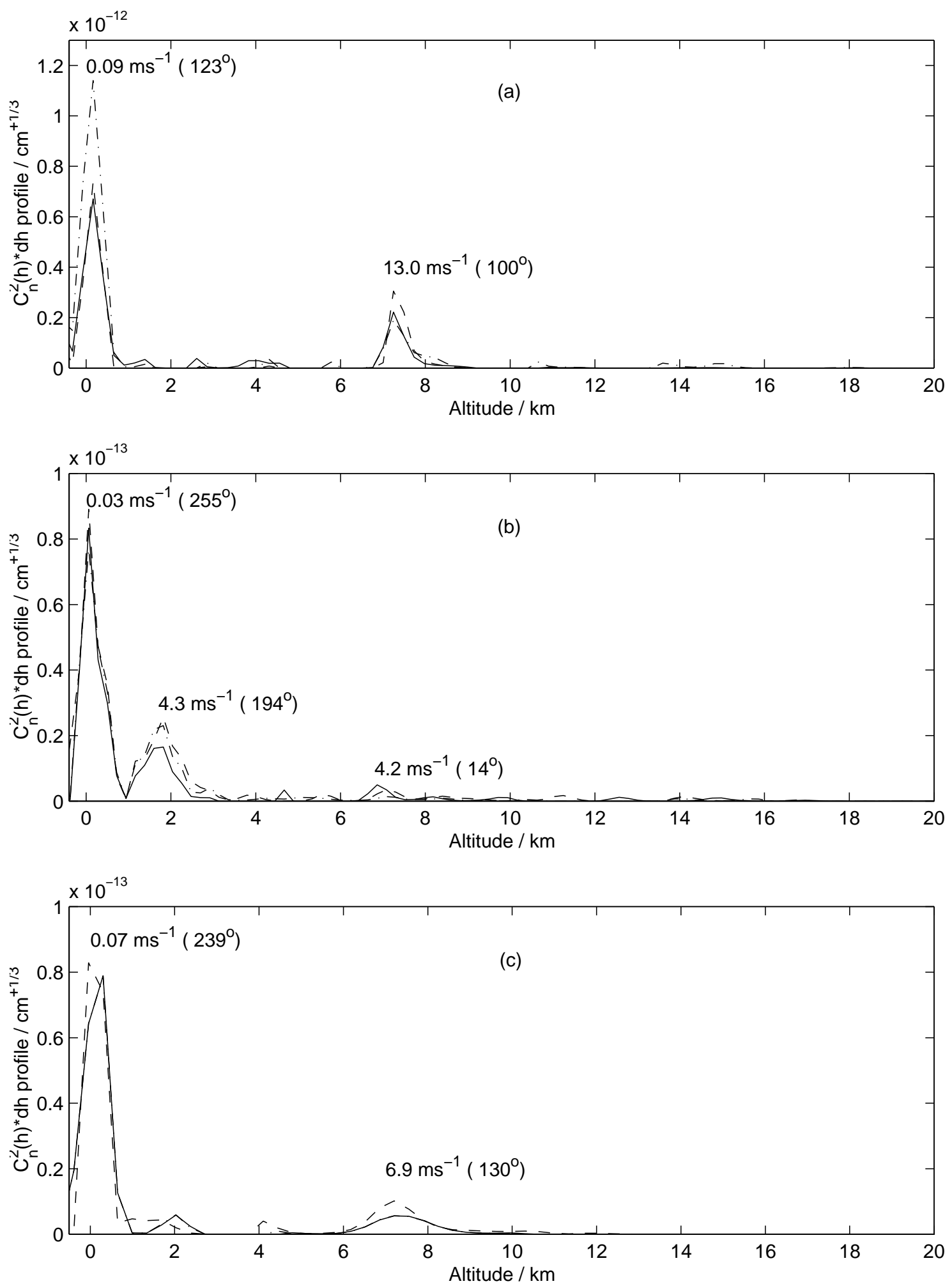

Fig. 11. Each figure shows three consecutive example $C_{n}^{2}(h)$ profiles from a) the La Palma observing site during November 1997, b) the Siding Spring observing site in Australia during January 1997 and c) the Calar Alto observing site in Spain during April 1997. The first profile is shown as a solid line, the second as a dotted line and the third as a dot-dashed line. Each profile was calculated from a 7 second block of data and each block was separated by around 10 seconds. The average speed and PA-direction of each dominant layer over the time period covered is also shown 

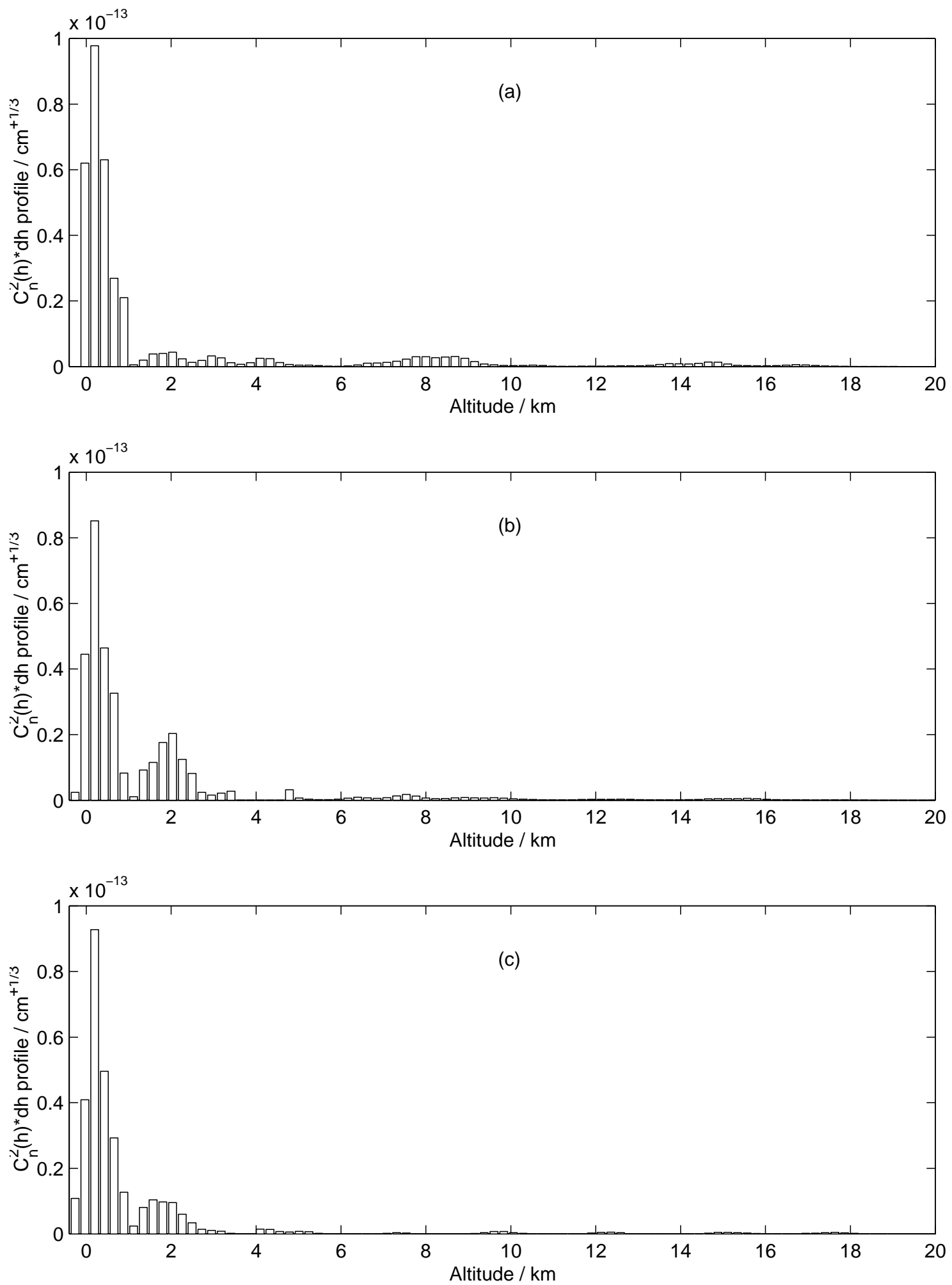

Fig. 12. a), b) and c) are three $C_{n}^{2}(h)$ profiles from the Siding Spring observing site in Australia during January 1997 showing longer-term variation. Each profile was calculated from a 4 minute block of data and each block was separated by 40 minutes 

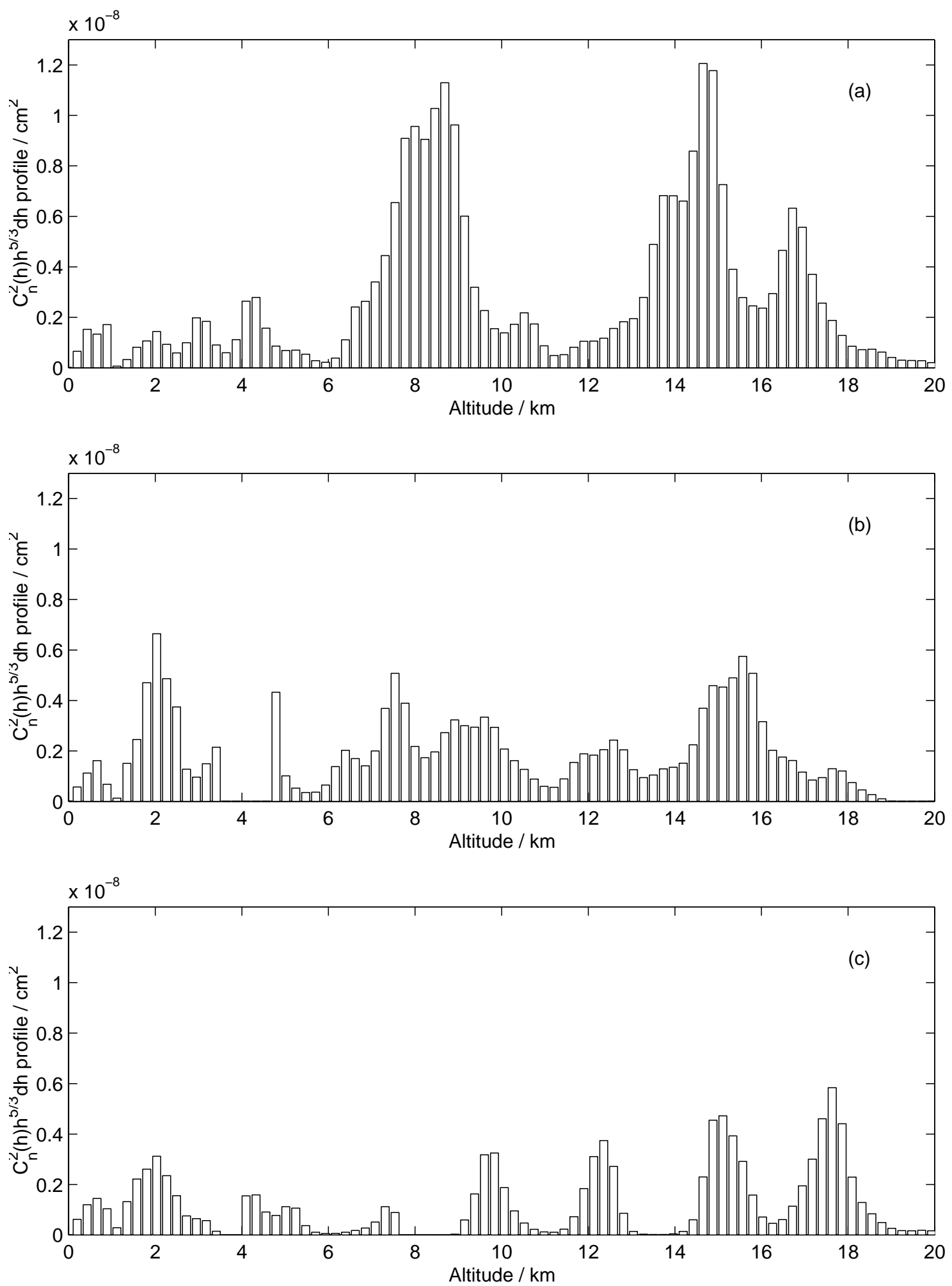

Fig. 13. As the previous figure but the $C_{n}^{2}(h)$ profile is now scaled by $h^{5 / 3}$ in order to illustrate the change in contribution to isoplanatic errors of the higher altitude layers 


\section{Conclusion}

In this paper we have described an implementation of generalised SCIDAR that provides estimates of the profiles of the refractive index structure function constant $C_{n}^{2}(h)$ and velocity profile $V(h)$. The technique has been verified using Monte Carlo propagation code and sample results from three locations presented. These show a fairly similar form, and as a general rule there are apparently just one or two dominant layers in addition to the inevitable layer in the region of the telescope itself.

The SCIDAR technique gives an excellent picture of the relative vertical distribution of optically significant turbulence; however, for the finite sampling levels here, there appears to be a tendency to obtain estimates of $C_{n}^{2}(h)$ that are too small, producing (for example) values of the Fried parameter $r_{0}$ that are too large. This could be due to spatial or temporal averaging effects, or to non-Rytov or non-Kolmogorov effects, since the theory assumes both Kolmogorov turbulence and the Rytov approximation. It would be preferable to simultaneously make a measurement (of, for example, the Fried parameter) that would allow a calibration of $C_{n}^{2}(h)$ to be made.

SCIDAR requires a medium-sized telescope, typically on the order of $1 \mathrm{~m}$, and suitable double stars of combined magnitude brighter than $m_{v} \approx 5$. Both of these facts are rather restrictive: for example, it would be desirable to have a $C_{n}^{2}(h)$ measurement device attached to a large telescope equipped with an adaptive optics (AO) feed to its instruments, so that the AO system could continuously operate in an optimised fashion such as being conjugated to a mean layer height. As an alternative to SCIDAR, single star techniques based on inverting the correlation of the scintillation may provide crude but adequate estimates of both $C_{n}^{2}(h)$ and $V(h)$.

Acknowledgements. We wish to thank Jean Vernin and Michel Tallon for the help and encouragement they have given us during our development of the SCIDAR technique and particularly in introducing us to the generalised SCIDAR method, and we also acknowledge Jean Vernin's contribution to this field over a number of years. We are also indebted to Nielson Roberty for his great contribution to our development of algorithms for the $C_{n}^{2}(h)$ inversion problem.

We wish to thank all of those observatory staff who helped in the preparation of our observations at the JKT at La Palma, the $40 \mathrm{inch}$ at Siding Spring and the $2.2 \mathrm{~m}$ at Calar Alto. In particular we wish to thank, Richard Wilson at RGO, John O'Byrne at Sydney University and Steve Beckwith at the Calar Alto Observatory for their continued support of, and practical help with, this project.

The observatory site at La Palma (and its sister on Tenerife, the Observatorio del Teide) is operated by the Instituto de Astrofsica de Canarias. The Isaac Newton Group (of which the JKT is a part) is operated by the Royal Greenwich Observatory on behalf of the UK Particle Physics \& Astronomy Research Council (PPARC) and the Nederlandse organisatie voor wetenschappelijk onderzoek (NWO).

The Visiting Astronomer, German-Spanish Astronomical Centre, Calar Alto, is operated by the Max-Planck-Institute for Astronomy, Heidelberg, jointly with the Spanish National Commission for Astronomy.

This work is supported by the Particle Physics \& Astronomy Council, UK.

\section{References}

Adcock M., Klückers V., Wooder N., Dainty J.C., 1996, in: Adaptive Optics, OSA Technical Digest Series 13, 200

Azouit M., Borgnino J., Vernin J., 1978, J. Opt. (Paris) 9, 291

Azouit M., Vernin J., 1980, J. Atmos. Sci. 37, 1550

Churnside J., Lataitis R., Lawrence R., 1988, Appl. Opt. 27, 2199

Fuchs A., Tallon M., Vernin J., 1994, Proc. SPIE 2222, 682

Martin H., 1987, PASP 99, 1360

Ochs G., Ting-i Wang, Lawrence R., Clifford S., 1976, Appl. Opt. 15, 2504

Racine R., Ellerbroek B., 1995, Proc. SPIE 2534, 248

Roddier C., Vernin J., 1977, Appl. Opt. 16, 2252

Rocca A., Roddier F., Vernin J., 1974, JOSA 64, 1000

Tyler G., 1992, in: Adaptive Optics for Large Telescopes, OSA Technical Digest Series 19, 8

Tyler G., 1994, JOSA A 11, 325

Skilling, Bryant, 1984, MNRAS 211, 111

Vernin J., Roddier F., 1973, JOSA 63, 270

Vernin J., Roddier F., 1975, C. R. Acad. Sci. Paris 280, B463

Vernin J., Barletti R., Ceppatelli G., Paterno L., Righini A., Speroni N., 1979, Appl. Opt. 18, 243

Vernin J., Azouit M., 1983, J. Opt. (Paris) 14, 131

Vernin J., Crochet M., Azouit M., Ghebrebrhan O., 1990, Radio Sci. 25, 953

Vernin J., Weigelt G., Caccia J., Muller M., 1991, A\&A 243, 553

Vernin J., Muñoz-Tuñôn C., 1992, A\&A 257, 811

Vernin J., Muñoz-Tuñôn C., 1994, A\&A 284, 311

Zavorotny V., 1992, Appl. Opt. 31, 7660 